\title{
An Integrated Signature Indexing Technique for Information Broadcasting over the Multi-level Wireless Channels
}

\author{
Garima Panwar \\ M.Tech. Student Dept. of \\ CS\&E, Ajay Kumar Garg \\ Engineering College \\ Ghaziabad (U.P.)
}

\author{
Vikas Goel \\ Assistant Prof. Dept. of \\ CS\&E, Ajay Kumar Garg \\ Engineering College \\ Ghaziabad (U.P.) India
}

\author{
Anil Kumar Ahlawat \\ Phd, Prof.\& Head Dept. of \\ MCA \\ Krishna Institute of \\ Engineering \&Technology \\ Ghaziabad (U.P.) India
}

\begin{abstract}
Mobile device has limited battery power, the minimization of the access time and tuning time for the broadcast data is an important problem. There have been many research efforts that focus on minimizing access time and tuning time by providing indexes on the broadcast data. This paper proposes two level energy efficient signatures indexing techniques over the multichannel to reduce power consumption for wireless broadcast. The two-level signature scheme is used for indexing the information frames. At the first level, the integrated signature is used to index the indexes about the information. At the second level a simple signature is used to index the indexes about the information.
\end{abstract}

Keywords- Wireless Environment, Multiple Broadcast Channels, Data Allocation, Broadcast Program, Index Information, integrated signature, simple signature.

\section{INTRODUCTION}

With the advent of wireless technologies, now people can access information anytime, anywhere on the move via wireless communications. However, different from traditional wired networks, some issues should be considered in the wireless environment. First, limited bandwidth of the wireless network and the energy needed for portable devices. Second, the environment is asymmetry, that is, from the power consumption point of view, sending data is more costly than receiving data for a portable computer. Due to these issues, the traditional request-response system [1] is no longer suitable for data dissemination in the wireless environment. Therefore, data dissemination in the wireless environment has become an interesting research problem [2][3][4].

In the broadcast-based system, the clients have to access data items sequentially in the broadcast channel.

The dissemination of information provided by broadcast-based information systems with a cost independent to the number of clients, which compensates for the limited bandwidth in the wireless environment. Moreover, just tuning the broadcast channel, clients can retrieve the broadcasted data, which results in a certain degree of energy saving. Therefore, data broadcast has become an attractive solution for information dissemination.

There are two factors affecting power consumption in broadcast system for mobile computers: (1) mobile computers can be switched between active mode (full power) and doze mode (power down) [5], and (2) receiving messages consumes less energy than sending messages. By switching between active and doze modes, power consumption is significantly reduced. Tune-in time and access time are two of the criterion for evaluating performance in the broadcast system-

- Access time The time elapsed from the moment an initial probe is made into the broadcast channel to the moment the desired data are acquired. This is the total time the clients must spend.

- $\quad$ Tuning time The time spent by the clients listening to the broadcast channel. There are two modes for the clients to operate. When the clients are listening to the data items in the broadcast channel, the CPU must operate in the active mode, which is costly for power consumption.

In the broadcast-based system, a broadcast program is needed which is used to construct the data sequence i.e. to the order of data items to broadcast. The main issue to generate a broadcast program is to minimize the average access time \& tune-in time for saving the limited resources and energy consumption in a mobile computing system. Many researcher has focused on generating broadcast programs for indexing the information on a single broadcast channel. However, few researchers are now attempting to trade off between access time and tune-in time on the multiple wireless channels environment.

\section{RELATED WORK}

There are several reasons for dividing data \& index over multiple channels instead of sending them over a single fast channel. Some are based on the constraints of the physical architecture. For example, clients may have heterogeneous communication capabilities, limiting the feasibility of single channel high-speed transmission [10]. Another is use of bandwidth conservation. Multiple multicast channels have been used to partition data in order to reduce total traffic [11]. Finally, some applications typically require only a small fraction of the total available bandwidth, so leftover bandwidth can be used by other applications.

Air indexing is widely adopted to conserve battery power in mobile devices. Several tree-based indexing techniques, like $\mathrm{B}+$ tree flexible indexing and distributed tree indexing, for broadcast channels are proposed [12]. However, these studies focus on one-dimensional indexes i.e. single channel indexes for equality-based queries. Traditional index techniques, such as hashing [12] and signature file [14], are also applied in air indexing, along with hybrid approach [15]. 


\section{SIGNATURE INDEXING ON SINGLE CHANNEL}

A signature is essentially an abstraction of the information stored in a record. It is generated by a specific signature function. By examining a data item's signature, of a data item, it is easy to say that the information frame. Since the size of a signature is much smaller than that of the data item itself, it is considerably more power efficient to index this signature along with the information, it is energy efficient solution examine signatures first instead of simply searching through all data items. In [9], three signature indexing schemes are proposed: simple signature, integrated signature, and multilevel signature.

\subsection{Simple Signature}

A signature frame is constructed by simply adding the signature data along with information frame. The signature frame is broadcasted before the corresponding information frame in Figure 1. When [9] a mobile user wants to retrieve information from the broadcast channel, they specify a query on mobile devices. A query signature query signature is generated based on the specified query. The channel tuned the device and uses query signature to compare with the frame signatures Received. When there a match found, the corresponding information frame is received by the mobile device for further checking in order to eliminate false drops. After checking further, If the frame is not a false drop, it will be retained in the result set. If the query signature does not match with a received frame signature, the device will switch into doze mode until the next signature frame arrives. If all of the frame signatures on the broadcast channel don't match with the query signature, the device will remain in doze mode for the most of the time \& while part of a broadcast cycle, thus saving a lot of energy. So it is a energy efficient solution for limited battery devices.

\begin{tabular}{|l|l|l|l|l|l|l|l|l|}
\hline SF1 & IF1 & SF2 & IF2 & SF3 & IF3 & $\ldots$ & SFn & IFn \\
\hline
\end{tabular}

Signature Frame (SF)

Information Frame (IF)

Fig. 1: Information Frame organization of signature indexing.

\subsection{Integrated Signature}

A generalized form of the simple signature scheme is to generate a integrated signature, called an integrated signature [9][10]. This integrated signature is for a group of one or more information frames, called a frame group. The integrated signature is broadcast before the frame group. In this scheme, any number of information frames may be indexed in a signature. For example, first signature indexes two information frames while the next signature indexes three information frames. The filtering procedure of the integrated scheme to identify the desired information frame is very similar to the simple signature scheme. This is also another solution of energy efficient problem.

\subsection{Multilevel Signature}

The multi-level signature scheme is a combination of the simple signature and integrated signature schemes .It consists of multiple levels of signatures. Signatures at the upper levels are integrated signatures and at the lowest level are simple signatures. In Figure 2. The gray signatures in the figure are integrated Signatures. An integrated signature indexes all of the information frames between itself and the next integrated signature of the same or at a higher level. The white signatures are frame signatures for the corresponding information frames. To reduce the false drop probability, the hashing functions used in generating the integrated signatures and simple signatures are different. To answer a query, a query signature is generated for each level of the signatures. After tuning into the broadcast channel, the corresponding query signatures are used to compare with different levels of signatures. If a signature fails in the comparison, the device switches to doze mode until a signature at the same or upper levels arrives. Otherwise, the device stays in active mode and continues the filtering process.

In this section, we have introduced the basic signature indexing technique these schemes are based on single channel. A signature is formed by hashing each field of a information into a random bit string and then superimposing together all the bit strings into a record signature. The number of collisions depends on how perfect the hashing function is and how many attributes an information frame has. Collisions in signature indexing occur when two or more information frames have the same signature. Usually the more attributes each information frame has, the more likely collisions will occur. Such collisions would translate into false drops, where clients download wrong information frames but with matching signatures. With basic signature indexing technique, signatures are broadcast together with information frames. The broadcast channel consists of signature buckets and information buckets. Each broadcast of a information bucket is preceded by a broadcast of the signature bucket, which contains the signature of the information frame. All signature buckets have equal length. Clients must shift through each broadcast bucket until the required information is found. The Information frame organization of signature indexing is illustrated in Fig. 2

\begin{tabular}{|l|l|l|l|l|l|l|l|l|l|l|}
\hline IS1 & SS1 & IF & IS 2 & SS2 & IF & IS n & SSn & IF n &.. & \\
\hline
\end{tabular}

Integrated signature (IS)

Simple signature (SS)

Fig. 2: Multi-level signature indexing 


\section{AN INTEGRATED SIGNATURE INDEXING ON MULTI-LEVEL WIRELESS CHANNEL}

Signature technique is very suitable in a wireless broadcasting environment for filtering information frame. Signature technique provides some advantages like applied to various type of information, this technique is good for multiple information retrieval and signature is very short compared to an information frame where access time will not be increase drastically. Integrated signature is constructed by collection of frames which are consecutive frames.

All of the information frames indexes by integrated signature between itself and the next integrated signature.

In this paper, we propose the two-level signature scheme. This scheme consists of two types of signatures: the simple signature and the integrated signature. A simple signature is constructed for each information frame. An integrated signature is constructed from a group of consecutive frames, called a frame group. In the two-level signature scheme, the first level signatures are integrated signatures and the second level signatures are simple signatures. Figure 3, the two-level signature scheme. The gray signatures in the figure are integrated signatures. An signature indexes all of the information frames between itself and the next integrated signature. (In the figure, an integrated signature indexes two information frames.) The white signatures are simple signatures for the corresponding information frames [7][8]. To reduce the chance of getting false drops, the hashing functions used in generating the integrated signatures and simple signatures are different. In figure, if desired data are available in index3 then client get offset value that particular information and switch to level2 to retrieve desired information frames.

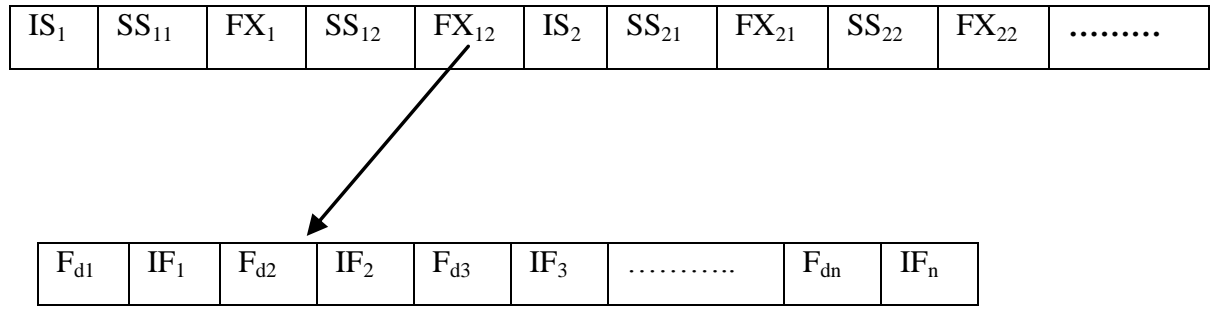

Fig. 3: Integrated Signature indexing on multi-level

\section{ALGORITHM}

Accessing information frames from integrated signature leve

begin

/ input:

$\mathrm{A}_{\mathrm{x}}=$ information frame that needs to be accessed;

$\mathrm{I}_{\mathrm{sz}}=$ the size of information frames;

$\mathrm{Ch}_{\text {no }}=$ data channel no over which data node $\mathrm{A}_{\mathrm{x}}$ is being broadcast;

Os = the time offset for retrieving the information frame $A_{x}$ over data channel; $\mathrm{Bc}=$ the no. of information Frames that must be broadcast over the data channel $\mathrm{Ch}_{\text {no. }}$, before the info. Frames $\mathrm{A}_{\mathrm{x}}$ is broadcasted again;

\section{Output:}

Location $=$ the current position of information frame $A_{x}$ over data channel $\mathrm{i}$ from index node/

Size-of-data-channel $=$ broadcast cycle $*$ size of information frame;

Period no $=$ current index position /data channel

Location $=$ size of data channel $*$ period no + offset

While ( current index position > Location)

\{periodno++;

Location $=$ size of data channel $*$ period no + offset

End

\section{PERFORMANCE ANALYSIS}

In signature indexing, there are various factors which are affecting the tune-in time and access time. For example, we must consider the number and the size of the signatures, the filtering capability of the signatures, the false drop probability of the signatures, and the initial probe time. A performance evaluation has to take these factors into account. The filtering capability and false drop probability may be controlled by the size of the signatures. On the other side, the initial probe time is related to the number of signatures interleaved with the information frames, and the access time and tune-in time are dependent on the number, size and false drop probability of the signatures. Therefore, in our analysis, the performance is estimated in terms of the number of packets.

Symbols and Parameters

$A_{t}:$ access time for signature channel.

$T_{t}$ : tuning time for signature channel.

$\mathrm{k}$ : number of information frames.

$\mathrm{n}$ : average no. of packets in an information frames.

1 : location of true drops.

$r$ : number of packets of signature.

$\mathrm{Q}_{\mathrm{s}}$ : selectivity of query.

$\mathrm{P}_{t}$ : the average initial probe time.

$\mathrm{S}_{\mathrm{i}}$ : integrated signature.

$\mathrm{S}_{\mathrm{s}}:$ simple signature. 
$\mathrm{D}_{\mathrm{i}}$ : data access from integrated signature.

$\mathrm{D}_{\mathrm{s}}$ : data access from simple signature.

$\mathrm{Os}_{\mathrm{i}}$ : offset on integrated signature.

$\mathrm{Os}_{\mathrm{s}}$ : offset on simple signature.

$\mathrm{F}_{\mathrm{di}}$ : false drop on integrated signature.

The access time for the signature indexing over the multilevel is:

\section{Access time $=$ Probe Time + Bcastcycle}

Now, Probe Time $=[\mathrm{r}+\mathrm{k} \times \mathrm{n}] / 2$

The length of a complete bcastcycle is:

$$
\text { Bcastcycle }=S_{i}+O_{s_{i}}+D_{i}+S_{s}+O_{S_{s}}+D_{s}
$$

\section{Access Time}

$$
A_{T}=P_{t}+S_{i}+O_{S_{i}}+D_{i}+S_{s}+O_{S_{S}}+D_{S}
$$

The tuning time for the signature indexing over the multilevel is:

Tuning time $=$ initial probe time + int. signature + int. offset + selectivity of query $* \mathrm{k} / \mathrm{l} *$ int. info. frames + int. false drops $*$ int info. frames + simp. signature + simp. Offset + simp. Info frames * selectivity of query + simp. Info frames * int. false drops - simp. Info. Frames *selectivity of query *int. false drops

$$
\begin{aligned}
\mathrm{T}_{\mathrm{T}}=\mathrm{P}_{\mathrm{t}}+\mathrm{S}_{\mathrm{i}}+\mathrm{O}_{\mathrm{S}_{\mathrm{i}}} & +\mathrm{Q}_{\mathrm{s}} \times \frac{\mathrm{K}}{\mathrm{l}} \times \mathrm{D}_{\mathrm{i}}+\mathrm{F}_{\mathrm{di}} \times \mathrm{D}_{\mathrm{i}}+\mathrm{S}_{\mathrm{s}}+\mathrm{O}_{\mathrm{S}_{\mathrm{s}}} \\
& +\mathrm{D}_{\mathrm{s}} \times \mathrm{Q}_{\mathrm{s}}+\mathrm{D}_{\mathrm{s}} \times \mathrm{F}_{\mathrm{di}}-\mathrm{D}_{\mathrm{s}} \times \mathrm{Q}_{\mathrm{s}} \times \mathrm{F}_{\mathrm{di}}
\end{aligned}
$$

Calculation: To calculate the access time and tune-in time on multichannel we are referring the 4.1 Eqn. Where parameters are given below:

$\mathrm{P}=128, \mathrm{n}=1000, \mathrm{~S}_{\mathrm{s}}=100, \mathrm{~S}_{\mathrm{i}}=280$,

$\mathrm{K}=4, \mathrm{l}=3, \mathrm{~A}=10240, \mathrm{P}_{\mathrm{s}}=0.01$,

Data $=9984$, Index $=256, r=2$,

$P_{f}^{i}=0.5$, Offset $=8$

$$
\begin{aligned}
& \mathbf{A}_{\mathrm{T} 1}=\frac{2+4 \times 1000}{2}+\frac{10240 \times 2}{4}+256+16+16+ \\
& 10240 \times 2+9984 \\
& =\quad 37,857
\end{aligned}
$$

$\mathbf{T}_{\mathbf{T 1}}=\frac{4+4000000}{2(2+4000)}+\frac{10240 \times 2}{4}+8+\frac{.01 \times 4}{3}+0.5 \times 256+$ $10240 \times 2+8+9984 \times .01+9984 \times 0.5-9984 \times$ $0.01 \times 0.5$

$$
=31,269
$$

We considering the following parameters, $\mathrm{A}_{2}=20480$, Index $=$ 512, Data $=19968, \mathrm{l}=3, \mathrm{r}=2, \mathrm{~K}=4, \mathrm{P}_{\mathrm{s}}=0.01$

$$
\begin{aligned}
& \mathbf{A}_{\mathbf{T} 2}=2001+\frac{20480 \times 2}{4}+512+8+8+20480 \times 2+ \\
& 19968 \\
& =73,697
\end{aligned}
$$

$$
\begin{aligned}
& \mathbf{T}_{\mathbf{T} 2}=499.75+10240+6.82+256+40960+199.68+ \\
& 9984+99.84 \\
& =\quad 62,046.41
\end{aligned}
$$

To calculate the access time and tune-in time on single channel with the Eqn. (1.3) and (1.4)

$$
\begin{aligned}
& \mathbf{A}_{\mathbf{T}}=\frac{\mathrm{K} \times \mathrm{n}^{2}+\mathrm{r}^{2}(\mathrm{~K}+1)+2 \times \mathrm{K} \times \mathrm{n} \times \mathrm{r}}{2(\mathrm{~K} \cdot \mathrm{n}+(\mathrm{K}+1) \mathrm{r})}+\mathrm{D}_{\mathrm{i}}+\mathrm{S}_{\mathrm{i}}+\mathrm{S}_{\mathrm{S}} \\
& \text { Eqn. }(1 \cdot 3) \\
& \mathbf{T}_{\mathbf{T}}=\mathrm{p}_{\mathrm{T}}+\mathrm{S}_{\mathrm{i}}+\left(\mathrm{I}-\left(\mathrm{P}_{\mathrm{S}} \times \frac{\mathrm{A}}{\mathrm{l}}\right) \cdot \mathrm{P}_{\mathrm{f}}^{\mathrm{i}} \cdot\left(\mathrm{K} \cdot \mathrm{r}+\mathrm{K} \cdot \mathrm{P}_{\mathrm{f}}^{\mathrm{S}} \cdot \mathrm{n}\right)+\right. \\
& \left(\mathrm{P}_{\mathrm{S}} \cdot \mathrm{A} / \mathrm{l}\right)\left(\mathrm{K} \cdot \mathrm{r}+\mathrm{l} \cdot \mathrm{n}+(\mathrm{K}-\mathrm{l}) \cdot \mathrm{P}_{\mathrm{f}}^{\mathrm{S}} \cdot \mathrm{n}\right)
\end{aligned}
$$

Eqn. (1.4)

$$
\begin{aligned}
& \mathbf{A}_{\mathrm{T} 1}=\frac{4 \times 1000000+5 \times 4+2 \times 4 \times 1000 \times 2}{2(4 \times 1000+5 \times 2)}+\frac{10240 \times 2}{4}+10240 \times \\
& 2+9984 \\
& =36,084.75 \\
& \mathbf{T}_{\mathbf{T 1}}=498.75+5120+(256-34) \times 0.5 \times(4 \times 2+4 \times \\
& 0.5 \times 1000)+\frac{0.01 \times 10240}{3} \times(4 \times 2+3 \times 1000+1 \times 8 \times \\
& \text { 1000) } \\
& =6,04,246 \\
& \mathbf{A}_{\mathrm{T} 2}=500.75+19968+\frac{20480 \times 2}{4}+20480 \times 2+10240+ \\
& =71,668.75 \\
& =6,95,058.75
\end{aligned}
$$

\section{COMPARISON \& ANALYSIS}

Using the formulae developed above, we compare the access time and tuning time on different channel. We assume that the selectivity of a query is $1 \%$ for the integrated and multi-level schemes. The false drop probability for the signature and integrated signatures can be calculated based on $\mathrm{m}$ and the number of bit strings superimposed $S_{s}$ and $S_{i}$. Since the integrated scheme is good for broadcast with similar information frames. Integrated signature on multi-level channels are better than the simple signature on single channel.

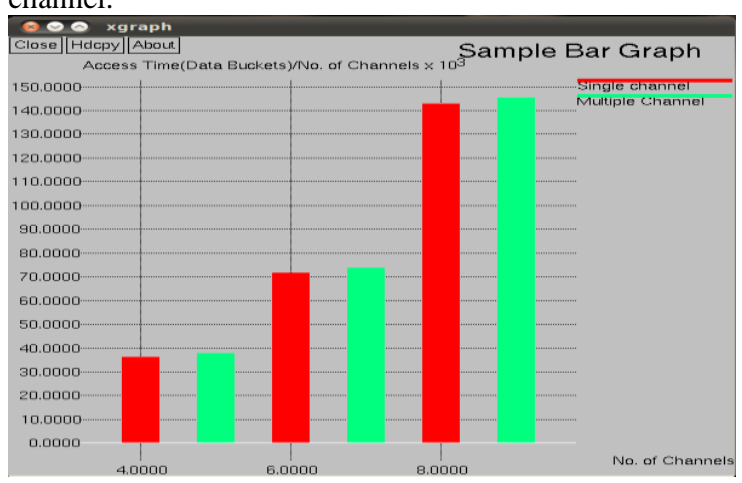

Fig. 4: Access Time (Data bucket) Vs no. of channels 


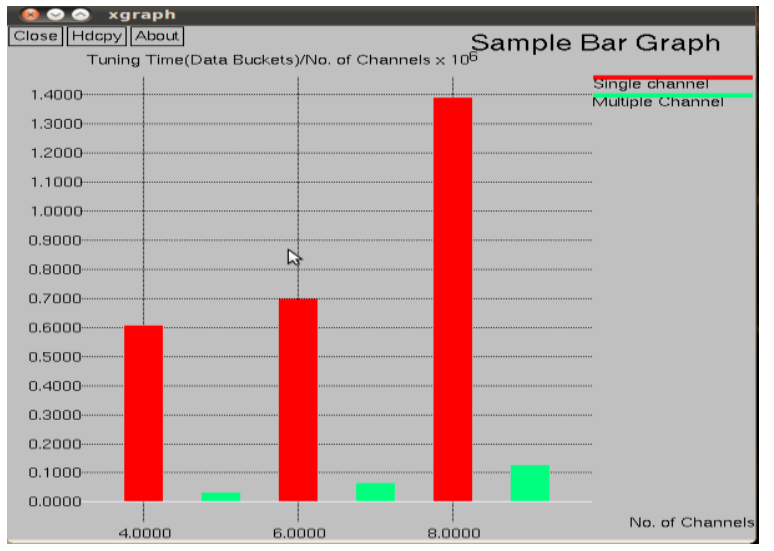

Fig. 5: Tuning Time (Data bucket) Vs no. of channels

Follow this (Fig. 4, and 5) access time is approximate equal over the single and multi-channel but tuning time drastically reduced over the multichannel. The integrated scheme is good for broadcast information frames. Integrated signature on multi-level channels is better than the simple signature on single channel.

\section{CONCLUSION}

Air indexing is an important technique that facilitates energy conservation of clients in wireless broadcast systems. Since most queries on broadcast information select only a small number of information frames, indexing is very effective in reducing the tune-in time. The integrated signature indexing is excellent for information filtering in information broadcast services. We can see that in Fig. 4 and 5, in this indexing scheme access time is approximate equal over the single and multi-channel but tuning time drastically reduced over the multi-channel. The integrated scheme is good for broadcast information frames. Integrated signature on multi-level channels is better than the simple signature on single channel. So, with the sake of reducing tuning time, we can conserve the battery consumption in wireless environment.

\section{REFERENCES}

[1] Tan, K.L and Ooi, B.C., Batch Scheduling for Demanddriven Servers in Wireless Environment.

[2] Alonso, R. and Korth, H., 1993.Database Systems in Nomadic Computing. In Proceedings of the 1993 ACM SIGMOD International Conference on Management of Data,388-392.

[3] Imielinski,T., Badrinath, B.R., 1993. Data Management for Mobile Computing. SIGMOD RECORD, 34-39.
[4] Pitoura, E. and Samaras, G., 1998. Data Management for Mobile Computing, Kluwer Academic Publishers.

[5] Ganguly, S. and Alonso, R. 1993. Query optimization in mobile environments, Technical Report No. LCSR-TR219, Department of Computer Science, Rutgers University.

[6] Amarmend, D., Aritsugi, M. and Kanamori, Y., 2006. An Air Index for Data Access over Multiple Wireless Broadcast Channels, Proceedings of the 22nd International Conference on Data Engineering (ICDE'06, IEEE.

[7] S. Jung, B. Lee, and S. Pramanik, "A tree-structured index allocation method with replication over multiple broadcast channels in wireless environments", IEEE Trans. on Knowledge and Data Engineering, March 2005, 17(3):311-325.

[8] Prabhakara, K., Hua, K., and Oh, J., 2000. Multi-level multi-channel air cache designs for broadcasting in a mobile environment. In Proc. 16th Int'l Conf. Data Eng., 167-176.

[9] Lee, W.C. and Lee, D., July 1996. Using signature techniques for information filtering in wireless and mobile environments, J. Distrib. Parallel Databases, vol. 4, no. 3 .

[10] Donahoo, M. J., Ammar, M. H., and Zegura, E. W. March 1999. Multiple-channel multicast scheduling for scalable bulk-data transport. In Proc. IEEE INFOCOM.

[11] Almeroth, K. C., Ammar, M. H., and Fei, Z., March 1998. Scalable delivery of web pages using cyclic besteffort (udp) multicast. In Proc. IEEE INFOCOM.

[12] Imielinski,T., Viswanathan, S. and Badrinath, B. R. May 1994. Energy efficiency indexing on air. In Proc. ACM SIGMOD International Conference on Management of Data (SIGMOD’94), 25-36.

[13] T. Imielinski, S. Viswanathan, and B. R. Badrinath, Data on air - organization and access. IEEE Trans. Knowledge and Data Engineering (TKDE), May-June 1997, 9(3):353-372.

[14] Hu, Q., Lee, W.C., and Lee, D., November 2-6 1999.Indexing techniques for wireless data broadcast under clustering and scheduling. In The Eighth International Conference on Information and Knowledge Management (CIKM'99.

[15] Q. Hu, W.-C. Lee, and D. Lee , March 2001, A hybrid index technique for power efficient data broadcast. Distributed and Parallel Databases (DPDB),9(2):151-177 